\title{
The Rectus Sheath Block (RSB) Analgesia Following Laparotomy Could Affect Malonidialdehyde (MDA) Concentrations in Benign Disease and Cancer
}

\author{
MAIJU RUOTTINEN ${ }^{1 *}$, VIIVI KUOSMANEN ${ }^{*}$, IINA SAIMANEN ${ }^{1 *}$, VIIVI KAARONEN ${ }^{1}$, DINA RAHKOLA ${ }^{1}$, \\ ANU HOLOPAINEN ${ }^{2}$, TUOMAS SELANDER ${ }^{3}$, HANNU KOKKI $^{4}$, MERJA KOKKI ${ }^{4}$ and MATTI ESKELINEN ${ }^{1}$ \\ ${ }^{1}$ Department of Surgery, Kuopio University Hospital and School of Medicine, \\ University of Eastern Finland, Kuopio, Finland; \\ ${ }^{2}$ Department of Clinical Chemistry, Eastern Finland Laboratory Center, \\ Kuopio University Hospital, Kuopio, Finland; \\ ${ }^{3}$ Science Service Center, Kuopio University Hospital, Kuopio, Finland; \\ ${ }^{4}$ Department of Anaesthesiology and Intensive Care, Kuopio University Hospital and School of Medicine, \\ University of Eastern Finland, Kuopio, Finland
}

\begin{abstract}
Background/Aim: The aim of the study was to compare the MDA (malonidialdehyde) plasma concentrations versus CAT (catalase)/NT (nitrotyrosine) plasma concentrations, patient satisfaction and pain score at rest/pressure to the wound area in laparotomy patients with rectus sheath block (RSB) analgesia. Patients and Methods: Initially, 56 patients were randomized to four groups; control group $(n=12)$, single-dose $(n=16)$, repeated-dose $(n=12)$ and continuous infusion $(n=16)$ RSB analgesia groups. The plasma concentrations of CAT, NT and MDA markers were measured just before, immediately after and $24 \mathrm{~h}$ after operation. Results: The RSB analgesia enhanced significantly patient satisfaction $(p=0.001)$. The plasma MDA decreased immediately after operation (POP1) and the postoperative decrease between the preoperative and the POP1 values in the MDA marker were statistically significant $(p<0.001)$. In linear mixed model, the time effect in both the single group and in the benign group in plasma NT biomarker was statistically significant ( $p=0.001, p=0.013$, respectively). The median plasma MDA concentrations $(\mathrm{ng} / \mathrm{ml})$ following surgery were significantly lower in patients with cancer versus patients
\end{abstract}

*These Authors contributed equally to this study.

Correspondence to: Matti Eskelinen, MD, Ph.D., School of Medicine, University of Eastern Finland, P.O. Box 100, FI-70029 KYS, Finland. Tel.: +358 17173311, Fax: +358 17172611, GSM: +358 400969444, e-mail: matti.eskelinen@kuh.fi

Key Words: Benign disease, cancer, lipid peroxidation, plasma malonidialdehyde, pain following surgery. with benign disease (589 vs. 852, $p=0.021)$. Jitterplots of the individual plasma NT versus plasma MDA showed that there was significant correlation in benign and cancer patients ( $r=0.347, p<0.001)$. Conclusion: Plasma MDA decreased significantly after operation in all patients and cancer patients had significantly lower MDA concentrations following surgery than patients with benign disease.

Reactive oxygen species (ROS) degrade polyunsaturated fatty acids (PUFAs) by lipid peroxidation forming malonidialdehyde (MDA) (1). MDA is one of the many ROS that cause oxidative stress in cells attacking the subcellular structures and forming covalent protein adducts (1). The production and plasma concentration of MDA can be measured and used as a biomarker to estimate the degree of oxidative stress and lipid peroxidation in human disease (2). MDA is a toxic substance reacting with deoxyadenosine in DNA and forming DNA adducts, which are mutagenic (3). A schematic representation of the relationship among the formation of ROS/Reactive nitrogen species (RNS) lipid peroxidation, nitrotyrosine (NT), catalase (CAT) and MDA is shown in five recent reviews $(1,4-7)$.

Our previous study showed that the control group of patients and the rectus sheath block (RSB) groups did not differ in individual pain scores (numeric rating scales, NRS) following surgery $(8,9)$. However, it seems that plasma CAT levels correlate NRS pain scores following surgery (10) and NT concentration does significantly correlate satisfaction for analgesia (9). There are seven large reviews in the literature available to compare ROS/RNS in clinical trials that investigated the impact of 


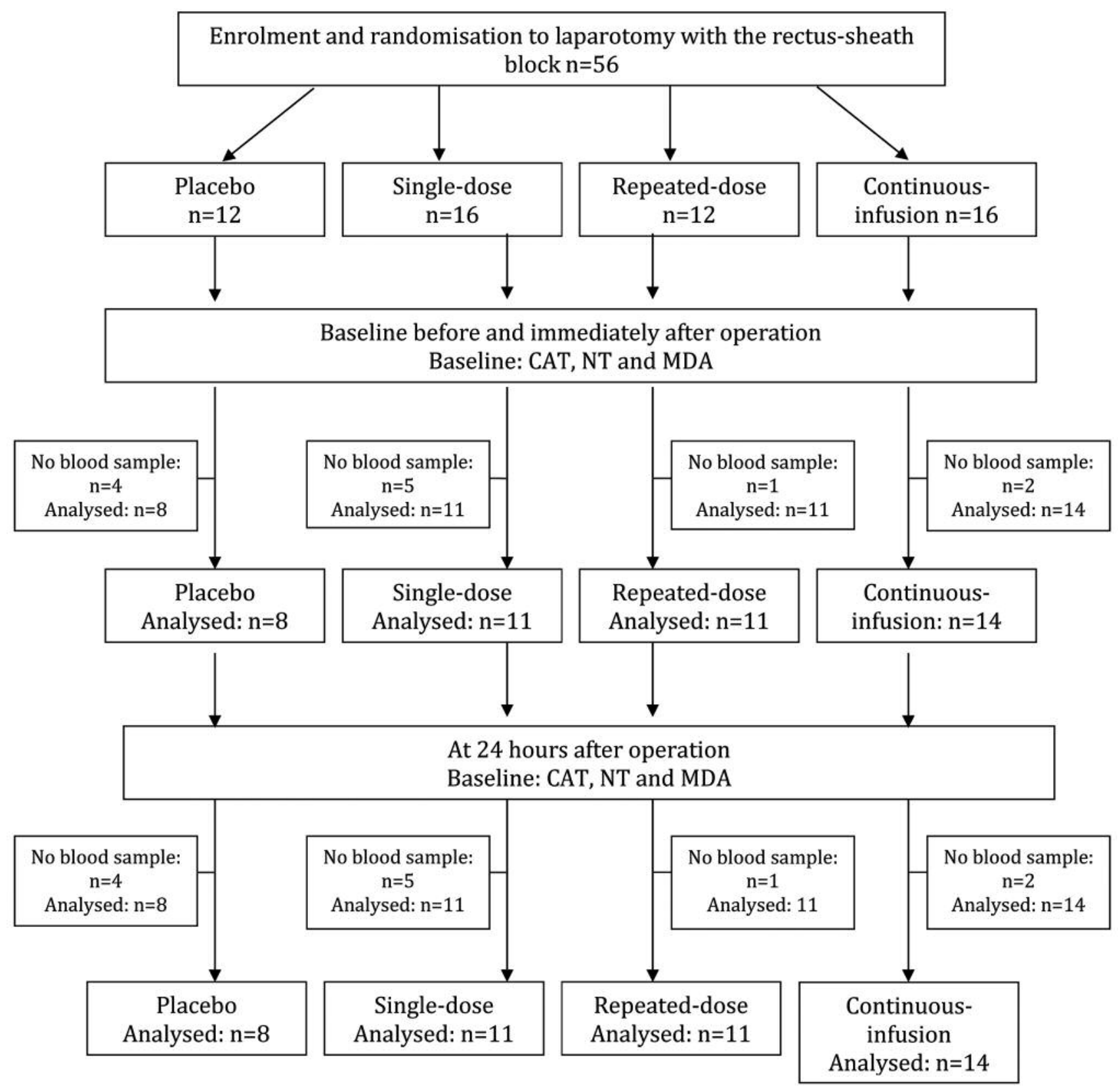

Figure 1. Study flowchart.

ROS/RNS in human disease $(1,4-7,11,12)$, however, there were no studies available on plasma MDA measurement in laparotomy with RSB. Therefore, the present study investigated the MDA plasma concentrations in patients with benign disease versus cancer following midline laparotomy. The main aim of this study was to study plasma MDA versus plasma CAT /NT concentrations, patient satisfaction and pain score at rest/pressure to the wound area in laparotomy patients with RSB.

\section{Patients and Methods}

The study was approved by the Ethics Committee of the Northern Savo Hospital District, Kuopio, Finland (DNRO 120/2011, November 11, 2011) and was registered in the EudraCT database (EudraCT number 2011-005136-25, Consort diagram, Figure 1) and in the ClinicalTrials.gov database (ClinicalTrials.gov Identifier: NCT02869841). It was conducted in accordance with the Declaration of Helsinki. Participants gave written consent after receiving verbal and written information. 
Table I. Clinical data of the four study groups. Data are mean (standard deviation) or number of cases.

\begin{tabular}{|c|c|c|c|c|c|}
\hline \multirow[b]{2}{*}{ Variable } & \multicolumn{4}{|c|}{ RSB group } & \multirow[b]{2}{*}{$p$-Value } \\
\hline & $\begin{array}{c}\text { Control } \\
n=8\end{array}$ & $\begin{array}{c}\text { Single } \\
\mathrm{n}=11\end{array}$ & $\begin{array}{c}\text { Repeated } \\
\mathrm{n}=11\end{array}$ & $\begin{array}{c}\text { Continuous } \\
\mathrm{n}=14\end{array}$ & \\
\hline Age, years & $62.6(14.3)$ & $60.8(12.6)$ & $63.3(10.8)$ & $58.0(10.1)$ & 0.74 \\
\hline Gender male/female & $4 / 4$ & $4 / 7$ & $2 / 9$ & $2 / 12$ & 0.22 \\
\hline Height, $\mathrm{cm}$ & $166.6(8.6)$ & $168.4(7.9)$ & $165.7(7.2)$ & $164.3(6.6)$ & 0.62 \\
\hline Weight, kg & $78.6(11.8)$ & $83.7(12.8)$ & $67.8(13.7)$ & $68.8(10.6)$ & 0.007 \\
\hline $\mathrm{BMI}, \mathrm{kg} / \mathrm{m}^{2}$ & $28.3(3.8)$ & $29.6(4.4)$ & $24.6(4.3)$ & $25.7(4.9)$ & 0.03 \\
\hline Time in the operative room, $\min$ & $229.4(113.4)$ & $274.9(148.4)$ & $235.7(112.0)$ & $279.7(178.5)$ & 0.85 \\
\hline Operative time, $\min$ & $209.6(141.2)$ & $221.8(156.4)$ & $154.4(95.0)$ & $253.3(168.9)$ & 0.55 \\
\hline \multicolumn{6}{|l|}{ Perioperative- } \\
\hline bleed, ml & $696(741)$ & $822(906)$ & $697(967)$ & $1,340(928)$ & 0.31 \\
\hline ASA $1 / 2 / 3 / 4$ & $0 / 6 / 2 / 0$ & $0 / 7 / 3 / 1$ & $0 / 5 / 6 / 0$ & $2 / 7 / 5 / 0$ & 0.43 \\
\hline $\mathrm{NRS}_{\mathrm{r}}$ & $2(1-6)$ & $2(0-4)$ & $2(0-3)$ & $1(0-4)$ & 0.41 \\
\hline $\mathrm{NRS}_{\mathrm{p}}$ & $5(3-9)$ & $5(1-9)$ & $3.5(1-7)$ & $4(0-7)$ & 0.42 \\
\hline $\mathrm{SFS}_{24}$ & $8(7-8)$ & $9(8-10)$ & $9.5(8-10)$ & $10(8.3-10)$ & 0.001 \\
\hline Length of the skin incision(s), $\mathrm{mm}$ & $27.2(6.6)$ & $24.4(7.8)$ & $24.2(7.9)$ & $29.7(7.3)$ & 0.31 \\
\hline Type of disease & & & & & 0.32 \\
\hline - Benign ( $\mathrm{n}=15)$ & 1 & 3 & 5 & 6 & \\
\hline - GI cancer $(n=9)$ & 3 & 2 & 2 & 2 & \\
\hline - Gyn cancer $(n=17)$ & 3 & 4 & 4 & 6 & \\
\hline - Other cancer $(n=3)$ & 1 & 2 & 0 & 0 & \\
\hline
\end{tabular}

GI: Gastrointestinal tract; Gyn: gynaecological; BMI: body mass index; ASA: American Society of Anesthesiologists physical status score; NRS: 11-point numeric rating scale $24 \mathrm{~h}$ after surgery; $\mathrm{NRS}_{\mathrm{r}}$ : NRS at rest; $\mathrm{NRS}_{\mathrm{p}}$ : NRS under $2 \mathrm{~kg}$ pressure to the wound area; $\mathrm{SFS}_{24}$ : satisfaction following surgery; RSB: rectal sheath block.

Operations were carried out at the Kuopio University Hospital, Kuopio, Finland between 2012 and 2015. The CONSORT flowchart of the study is presented in Figure 1. The study design was a prospective, randomised, clinical trial with four parallel groups. The patients with midline laparotomy were randomized into the control group or into one of the three active groups; single-dose, repeateddose or continuous infusion RSB analgesia groups. The study patients had intravenous oxycodone pumps as the patient-controlled analgesia (PCA). The randomisation list was generated by a computer (13), a sealed enveloped method was used for blinding and randomisation was performed preoperatively. The patients in the control group had no RSB-catheters inserted. However, the control patients were blinded using the similar wound dressing as the patients in the active groups. The design of this study, the inclusion and exclusion criteria and the RSB analgesia procedure is fully described in our earlier reports (14-16).

The primary outcome measures were the plasma concentrations of CAT, NT and MDA biomarkers measured at three time points with high-sensitivity assays: before (PRE), immediately after (POP1) and $24 \mathrm{~h}$ postoperatively (POP2) in the control group versus three active groups. The patient satisfaction, the overall pain at rest $\mathrm{NRS}_{\mathrm{r}}$ and at $2 \mathrm{~kg}$ pressing $\mathrm{NRS}_{\mathrm{p}}$ were surveyed and filed on a 11point numeric rating scale as shown previously (17).

EDTA-blood samples were taken at the pre-specified time-points and centrifuged at $1,000 \times g$ for $15 \mathrm{~min}$. Plasma was separated and stored frozen at $-70^{\circ} \mathrm{C}$ until analyzed. The plasma CAT assays were performed using enzyme-linked immunosorbent assay ELISA Kit (Cloud-Clone Corporation, Katy, TX, USA). The plasma NT assays were performed using competitive enzyme immunoassay OxiSelect ${ }^{\mathrm{TM}}$
Nitrotyrosine ELISA Kit (STA-305, Cell Biolabs Inc, San Diego, CA, USA). Plasma MDA concentrations were determined by using MDA (Malondialdehyde) ELISA Kit (E-EL-0060, Elabscience, USA). The manufacturer's intra-assay and the inter-assay Cvs were $5.6 \%$ and $6.28 \%$, respectively.

Data are presented as means and standard deviations or frequencies and percentaces, where appropriate. Differences in baseline characteristics between groups were tested by the Fisher's exact test and in the case of continuous data, the analysis was performed by the Kruskall-Wallis $t$-test. Group differences at three time points were tested by the Wilcoxon signed rank test and the Kruskall-Wallis-test. The plasma CAT, NT and MDA concentrations and differences in the patients with benign diseases and cancer were tested by the Mann-Whitney $U$-test. Linear mixed effect (LME) model was used to test the interaction group time effect. In LME analysis plasma concentrations were log transformed. The results of the laboratory measurements are presented as median concentrations with interquartile range as distributions were right-skewed. $p$-Values under 0.05 were considered statistically significant. The Pearson's method was used to test for correlation for plasma NT concentrations versus MDA concentrations.

\section{Results}

The control patients and three RSB groups were quite equal in terms of perioperative data; age, gender, height, weight, body mass index, time in the operative room, operative time, perioperative bleed, American Society of Anesthesiologists, 
Table II. Plasma catalase (CAT), nitrotyrosine (NT) and malonidialdehyde (MDA) concentrations in the four study groups. Plasma concentrations were measured before (PRE), immediately after (POP1) and $24 \mathrm{~h}$ after (POP2) surgery. Median (interquartile range) concentrations are shown. The Kruskall-Wallis test was used.

\begin{tabular}{|c|c|c|c|c|c|}
\hline \multirow[b]{2}{*}{ Marker } & \multicolumn{4}{|c|}{ RSB group } & \multirow[b]{2}{*}{$p$-Value } \\
\hline & Control & Single & Repeated & Continuous & \\
\hline CAT (pg/ml) & & & & & 0.506 \\
\hline PRE & $0.95(0.67-2.36)$ & $0.74(0.45-1.58)$ & $0.61(0.41-1.09)$ & $0.61(0.43-1.17)$ & 0.407 \\
\hline POP1 & $1.36(0.89-3.71)$ & $1.89(0.78-2.93)$ & $1.53(0.78-2.17)$ & $1.39(0.84-2.03)$ & 0.907 \\
\hline POP2 & $1.17(0.58-1.65)$ & $0.86(0.60-1.73)$ & $1.15(0.77-1.50)$ & $1.06(0.83-1.92)$ & 0.891 \\
\hline NT (pg/ml) & & & & & 0.042 \\
\hline PRE & $5.47(3.03-6.01)$ & $7.15(5.84-8.81)$ & $5.28(4.00-8.74)$ & $6.25(5.09-8.78)$ & 0.128 \\
\hline POP1 & $4.03(2.98-5.93)$ & $7.74(5.96-9.16)$ & $5.54(3.21-7.21)$ & $5.96(4.30-8.43)$ & 0.127 \\
\hline POP2 & $4.39(3.24-7.55)$ & $4.58(3.41-5.87)$ & $4.70(4.15-5.51)$ & $6.37(4.55-7.31)$ & 0.277 \\
\hline MDA (ng/ml) & & & & & 0.448 \\
\hline PRE & $642(480-1241)$ & $872(670-1132)$ & 795 (685-907) & $983(688-1329)$ & 0.478 \\
\hline POP1 & $541(321-1165)$ & $753(576-801)$ & $555(430-698)$ & $638(525-909)$ & 0.484 \\
\hline POP2 & $480(413-946)$ & $651(533-836)$ & $577(343-799)$ & $601(373-784)$ & 0.767 \\
\hline
\end{tabular}

Linear mixed model $p$-values for the interaction time group are in bold. Time effect in linear mixed model in plasma NT in single dose group was statistically significant $(p=0.001)$.

physical status, classification, length of skin incision, type of disease, pain at rest and pain under $2 \mathrm{~kg}$ pressure to the wound area (Table I). The RSB study cohort consisted of 15 patients with benign disease, nine patients with gastrointestinal cancer, 17 patients with gynaecological cancer and three patients with other malignancy (Table I). The RSB analgesia enhanced significantly the median $\mathrm{SFS}_{24}$ scores in the following order; control, single dose, repeated dose and continuous dose group (8; $9 ; 9.5$ and 10 , respectively, $p=0.001$, Table I).

There were no statistically significant differences detected in the CAT, NT and MDA concentrations between the control group patients and the three active groups preoperatively and following surgery. Although, the patients in the single dose group had a trend for higher median NT levels preoperatively and immediately after operation (POP1) compared to the control group, repeated dose and continuous dose groups (median plasma concentrations $\mathrm{pg} / \mathrm{ml}, 7.15 / 7.74$ versus 5.47/4.03 and 5.28/5.54 and 6.25/5.96, respectively, Table II). Time effect in the linear mixed model in plasma NT (Single group) was statistically significant $(p=0.001)$. The patients in the control group had a trend for lower median MDA concentrations preoperatively and following surgery compared to the active RSB groups (median plasma MDA concentrations $\mathrm{ng} / \mathrm{ml}$, control group; 642/541/480 versus single dose; 872/753/651 and repeated dose; 795/555/577 and continuous infusion; 983/638/601; Table II).

The postoperative alteration of plasma CAT, NT and MDA marker concentrations in the groups combined are shown in Table III. There were statistically significant increases in the median plasma concentrations of CAT preoperatively (PRE) and immediately after operation (POP1) $(p<0.001$, Table III). Then the median plasma concentrations of CAT marker decreased $24 \mathrm{~h}$ postoperatively (POP2) and the decrease between the POP1 and POP2 values in the CAT marker was statistically highly significant $(24 \%$ decrease, $p=0.001$, Table III). The median plasma concentrations of the NT marker decreased $24 \mathrm{~h}$ postoperatively (POP2) and the decrease between the POP1 and POP2 concentrations in the NT were statistically significant ( $17 \%$ decrease, $p=0.041$, Table III). There was a statistically significant decrease in the median plasma concentrations of MDA preoperatively and immediately after operation ( $p<0.001$, Table III). Also, the median plasma concentrations of MDA decreased $24 \mathrm{~h}$ following surgery (POP2) and the decrease between the POP1 and POP2 concentrations in MDA were statistically significant ( $p=0.047$, Table III).

The median (interquartile range) plasma concentrations of CAT, NT and MDA do not differ significantly between the benign and cancer patients preoperatively (Table IV). Patients in the cancer group had a trend for lower median NT levels compared to benign patients (Table IV). However, there was a statistically significant difference in median NT concentrations between benign and cancer patients immediately following surgery (POP1) ( $p=0.008$, Table IV). In addition, time effect in the linear mixed model in plasma NT (Benign patients) was statistically significant $(p=0.013)$. There was a statistically significantly higher MDA concentration immediately after surgery in the benign group of patients than in the cancer group ( $852 \mathrm{ng} / \mathrm{ml}$ versus 589 $\mathrm{ng} / \mathrm{ml}$, respectively, Table IV). 
Table III. Alteration of plasma catalase (CAT), nitrotyrosine (NT) and malonidialdehyde (MDA) concentrations following surgery in all groups combined (all patients). Plasma concentrations were measured before $(P R E)$, immediately after (POP1) and 24 h postoperatively (POP2). Median (interquartile range) concentrations are shown.

\begin{tabular}{lccr}
\hline Marker & All patients & Alteration & $p$-Value* \\
\hline CAT(pg/ml) & & & \\
PRE & $0.66(0.48-1.17)$ & & \\
POP1 & $1.39(0.84-2.43)$ & PRE vs. POP1 & $<0.001$ \\
POP2 & $1.03(0.70-1.57)$ & POP1 vs. POP2 & 0.001 \\
NT(pg/ml) & & & \\
PRE & $6.02(4.78-8.16)$ & & \\
POP1 & $5.96(4.14-8.03)$ & PRE vs. POP1 & 0.361 \\
POP2 & $4.96(4.12-6.53)$ & POP1 vs. POP2 & 0.041 \\
MDA(ng/ml) & & & \\
PRE & $870(625-1118)$ & & \\
POP1 & $620(432-860)$ & PRE $v s$. POP1 & $<0.001$ \\
POP2 & $595(410-831)$ & POP1 vs. POP2 & 0.047 \\
\hline
\end{tabular}

*Wilcoxon signed-rank test.

The median (interquartile range) plasma concentrations of CAT do not correlate significantly to NT concentrations in benign and cancer patients (data not shown). Figure 2 shows a jitterplot of the plasma NT concentrations versus plasma MDA concentrations in benign and cancer patients $(r=0.347$, $p<0.001)$.

\section{Discussion}

The MDA plasma concentrations versus $\mathrm{CAT} / \mathrm{NT}$ plasma concentration, patient satisfaction and NRS pain score at rest $\left(\mathrm{NRS}_{\mathrm{r}}\right)$ and NRS pain score at $2 \mathrm{~kg}$ pressure to the wound area $\left(\mathrm{NRS}_{\mathrm{p}}\right)$ in midline laparotomy patients with RSB analgesia is unknown. The original study hypothesis was that RSB analgesia could lower pain and enhance satisfaction in benign and cancer patients. The main aim of this study was to investigate the relation between the MDA biomarker concentration and CAT/NT concentration and RSB analgesia in patients with midline laparotomy. Interestingly, plasma MDA concentrations decreased significantly after operation in all study patients. However, no statistically significant differences were detected between MDA concentration and pain scores $\mathrm{NRS}_{\mathrm{r}} / \mathrm{NRS}_{\mathrm{p}}$ and patients' satisfaction in the control group and three RSB study groups.

In the present study, patients in the cancer group had a trend for lower median MDA concentration compared to the benign group patients and there was a statistically significant difference in median MDA concentration between benign and cancer patients immediately following surgery (POP1). In addition, the postoperative alteration (PRE versus POP1) of MDA concentrations in all groups combined (all patients) was statistically significant in the single dose group had a
Table IV. Plasma catalase (CAT), nitrotyrosine (NT) and malonidialdehyde (MDA) concentrations in the patients with benign diseases ( $n=15)$ and in those with cancer $(n=29)$. Plasma concentrations were measured before (PRE), immediately after (POP1) and 24 hours postoperatively (POP2). Median (interquartile range) concentrations are shown. Mann-Whitney U-test is used.

\begin{tabular}{lccc}
\hline Marker & Benign & Cancer & $p$-Value \\
\hline CAT(pg/ml) & & & $\mathbf{0 . 7 1 8}$ \\
PRE & $0.62(0.41-1.17)$ & $0.73(0.48-1.30)$ & 0.407 \\
POP1 & $1.09(0.78-2.50)$ & $1.46(1.13-2.36)$ & 0.304 \\
POP2 & $0.83(0.75-1.65)$ & $1.12(0.68-1.50)$ & 0.593 \\
NT(pg/ml) & & & $\mathbf{0 . 0 1 6}$ \\
PRE & $6.83(5.05-8.32)$ & $5.67(4.10-8.10)$ & 0.250 \\
POP1 & $7.58(5.96-9.71)$ & $5.28(3.41-6.44)$ & 0.008 \\
POP2 & $5.75(4.35-6.73)$ & $4.69(4.09-6.27)$ & 0.308 \\
MDA(ng/ml) & & & $\mathbf{0 . 3 5 3}$ \\
PRE & $920(716-1326)$ & $868(571-1037)$ & 0.211 \\
POP1 & $852(614-955)$ & $589(392-730)$ & 0.021 \\
POP2 & $697(407-868)$ & $560(411-758)$ & 0.383 \\
\hline
\end{tabular}

Linear mixed model $p$-values for the interaction time group are in bold. Time effect in linear mixed model in plasma NT in benign group patients was statistically significant $(p=0.013)$.

trend for higher plasma NT concentrations preoperatively (PRE) and immediately following surgery (POP1) compared to other study groups. We have earlier reported that RSB analgesia does significantly alter the oxidative stress markers SOD1/CAT concentrations (increase) and NT concentrations (decrease) immediately after operation, but SOD1/CAT marker concentrations drops back $24 \mathrm{~h}$ following surgery (8$10,18)$. Interestingly, in the present study we found that the time effect in LME analysis in plasma NT biomarker both in the single group patients and in benign patients group was statistically significant ( $p=0.001$ and $p=0.013$, respectively).

ROS degrade polyunsaturated fatty acids by lipid peroxidation forming MDA (1). PUFAs located in cellular membranes can readily react with free radicals and undergo lipid peroxidation. Peroxidation of PUFAs on cell membranes alters cell membrane transport mechanism, membrane channel function and membrane receptor interactions (1). In earlier studies MDA biomarker has been used to show the connection between oxidative stress and chronic obstructive pulmonary disease (COPD) (19), lumbar disc degeneration (LDD) (20), myocardial ischemia (21) and diabetes mellitus (DM) (22). A review by Sardaro et al. (6) concluded that association between the most frequent inflammatory, potentially malignant diseases is well established. Lu et al. 2018 (19) studied the effect of oligomeric proanthocyanidin (OPC) (23) on the oxidative stress and lung function in COPD patients. The results showed that OPC supplementation significantly reduced the plasma SOD and MDA biomarker concentrations and the OPC supplementation was effective in increasing the antioxidant 


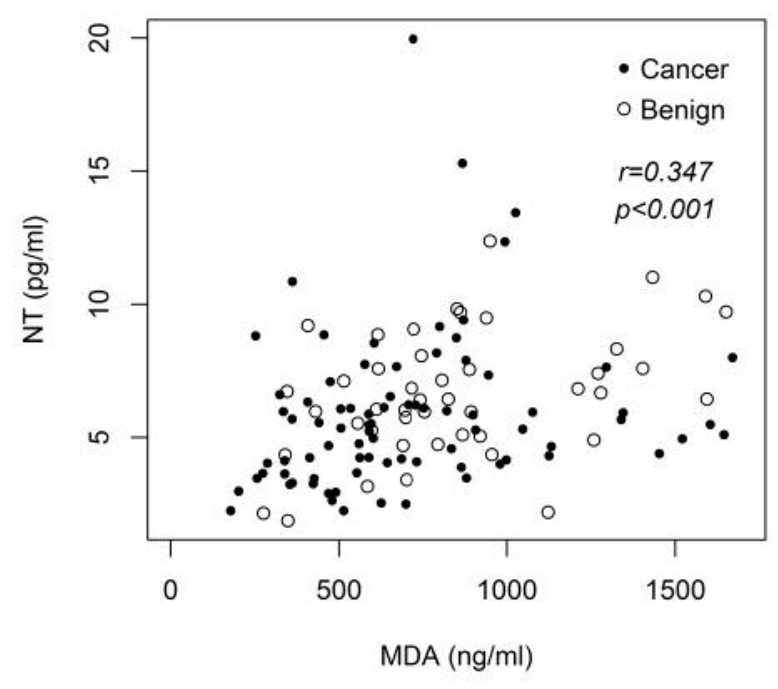

Figure 2. Jitterplot of plasma nitrotyrosine (NT) concentration versus plasma malonidialdehyde (MDA) concentration in benign and cancer patients $(r=0.347, p<0.001)$.

capacity of patients with COPD. Bakirezer et al. (20) evaluated serum MDA concentrations in patients with LDD. Serum MDA concentrations were significantly higher in the LDD patient group compared to the control group $(p=0.025)$. Oxidative stress seems to play a role in LDD patients. Dogaru et al. (21) studied the effect of mofettes which are gases resulting from natural post-volcanic emnations that are found in certain areas of Romania. They used Wistar-Bratislava rats divided into three groups: 1, control group; 2, isoproterenolinduced myocardial ischemia group; 3 , isoproterenol-induced myocardial ischemia+mofette group. MDA was used as a biomarker of oxidative stress, however, no statistically significant difference in collected blood MDA concentrations between groups 2 and 3 was found. Tsounapi et al. (22) evaluated OS alterations in the bladder of type 2 diabetes rat model and whether treatment could be beneficial for the bladder. They found that the level of MDA in the bladder was significantly higher in the DM group compared to all other study groups. In addition, immunochemistry showed that DM induced a moderated to strong expression of MDA in the DM group compared to other study groups.

The most important finding is a significant correlation between RSB analgesia and patients satisfaction $24 \mathrm{~h}$ following surgery in benign and cancer patients. Plasma MDA concentrations decreased significantly after operation in all patients and interestingly, the cancer patients had significantly lower MDA concentrations following surgery than patients with benign disease. Interestingly, in the present study we found that the time effect in the LME analysis in plasma NT biomarker both in the single group patients and in benign patients was statistically significant.

\section{Conflicts of Interest}

The Authors report no conflicts of interest or financial ties to disclose. The Authors alone are responsible for the content and writing of this article.

\section{Authors' Contributions}

All Authors have met all of the following four criteria: 1 . Substantial contributions to the conception or design of the work or the acquisition, analysis, or interpretation of data for the work, 2. Drafting the work or revising it critically for important intellectual content, 3. Final approval of the version to be published, 4. Agreement to be accountable for all aspects of the work in ensuring that questions related to the accuracy or integrity of any part of the work are appropriately investigated and resolved.

\section{Acknowledgements}

The study was funded by the Heikki, Aino and Aarne Korhonen foundation and the EVO-funding of the Kuopio University Hospital, Finland.

\section{References}

1 Akyol O, Zoroglu SS, Armutcu F, Sahin S and Gurel A: Nitric oxide as a physiopathological factor in neuropsychiatric disorders. In Vivo 18: 377-390, 2004. PMID: 15341194.

2 Del Rio D, Stewart AJ and Pellegrini N: A review of recent studies on malondialdehyde as toxic molecule and biological marker of oxidative stress. Nutr Metab Cardiovasc Dis 15: 316328, 2005. PMID: 16054557. DOI: 10.1016/j.numecd. 2005.05 .003

3 Marnett LJ: Lipid peroxidation-DNA damage by malondialdehyde. Mutat Res 424: 83-95, 1999. PMID: 10064852. DOI: 10.1016/s0027-5107(99)00010-x

4 Calabrese V, Boyd-Kimball D, Scapagnini G and Butterfield DA: Nitric oxide and cellular stress response in brain aging and neurodegenerative disorders: The role of vitagenes. In Vivo 18: 245-267, 2004. PMID: 15341181.

5 Seyidova D, Aliyev A, Rzayev N, Obrenovich M, Lamb BT, Smith MA, de la Torre JC, Perry G and Aliev G: The role of nitric oxide in the pathogenesis of brain lesions during the development of Alzheimer's disease. In Vivo 18: 325-333, 2004. PMID: 15341188.

6 Sardaro N, Della Vella F, Incalza MA, DI Stasio D, Lucchese A, Contaldo M, Laudadio C and Petruzzi M: Oxidative stress and oral mucosal diseases: An overview. In Vivo 33: 289-296, 2019. PMID: 30804105. DOI: 10.21873/invivo.11474

7 von Woedtke Th, Schmidt A, Bekeschus S, Wende K and Weltmann K: Plasma Medicine: A field of applied redox biology. In Vivo 33: 1011-1026, 2019. PMID: 31280189. DOI: 10.21873/invivo. 11570

8 Saimanen I, Kärkkäinen J, Selander T, Purdy M, Kokki M, Kokki $\mathrm{H}$ and Eskelinen M: Plasma catalase in relation to pain following midline laparotomy: a prospective study of patients with benign diseases and patients with cancer. Anticancer Res 38: 6479-6484, 2018. PMID: 30396975. DOI: 10.21873/ anticanres.13011 
9 Kuosmanen V, Saimanen I, Rahkola D, Kärkkäinen J, Selander T, Purdy M, Kokki H, Kokki M and Eskelinen M: Rectus sheath block (RSB) analgesia could enhance significantly the patient satisfaction following midline laparotomy in benign disease and in cancer: a prospective study with special reference to nitrosative stress marker nitrotyrosine (NT) plasma concentrations. Anticancer Res 39: 1383-1389, 2019. PMID: 30842172. DOI 10.21873/anticanres.13252

10 Saimanen I, Kuosmanen V, Kärkkäinen J, Selander T, Aspinen $\mathrm{S}$, Holopainen $\mathrm{A}$, Rantanen $\mathrm{T}$ and Eskelinen $\mathrm{M}$ : Cholecystectomy patients with high plasma level of catalase have significantly lower analgesia requirement: A prospective study of two different cholecystectomy techniques with special reference to patients with cancer. Anticancer Res 38: 5417-5422, 2018. PMID: 30194197. DOI: 10.21873/anticanres.12872

11 Arsalani-Zadeh R, Ullah S, Khan S and MacFie J: Oxidative stress in laparoscopic versus open abdominal surgery: A systematic review. J Surg Res 169: 59-68, 2011. PMID: 21492871. DOI: 10.1016/j.jss.2011.01.038

12 Yiannakopoulou ECh, Nikiteas N, Perrea D and Tsigris C: Effect of laparoscopic surgery on oxidative stress response: systematic review. Surg Laparosc Endosc Percutan Tech 23: 101-108, 2013. PMID: 23579502. DOI: 10.1097/SLE.0b013e3182827b33

13 Randomization plan generators. Available at: http:// www.randomization.com

14 Purdy M, Kokki M, Anttila M, Aspinen S, Juvonen P, Selander T, Kokki H, Pulkki K and Eskelinen M: Does the post-surgery placement of rectus sheath block analgesia alter the oxidative stress biomarker 8-OHdG concentrations: A randomised trial of patients with cancer and benign disease. Cancer Genomics Proteomics 13: 239-244, 2016. PMID: 27107066.

15 Purdy M, Kokki M, Anttila M, Aspinen S, Juvonen P, Korhonen R, Selander T, Kokki H and Eskelinen M: Does the rectus sheath block analgesia reduce the inflammatory response biomarker IL$1 \mathrm{ra}$, IL-6, IL-8, IL-10 and IL-1 $\beta$ concentrations following surgery? A randomised clinical trial of patients with cancer and benign disease. Anticancer Res 36: 3005-3011, 2016. PMID: 27272818

16 Purdy M, Kärkkäinen J, Kokki M, Anttila M, Aspinen S, Juvonen P, Kokki H, Pulkki K, Rantanen T and Eskelinen M: Does rectus sheath block analgesia alter levels of the oxidative stress biomarker glutathione peroxidase: A randomised trial of patients with cancer and benign disease. Anticancer Res 37: 897902, 2017. PMID: 28179349. DOI: 10.21873/anticanres.11396

17 Kuosmanen V, Ruottinen M, Rahkola D, Saimanen I, Kaaronen V, Selander T, Purdy M, Kokki H, Kokki M and Eskelinen M: Brief pain inventory (BPI) health survey after midline laparotomy with the rectus sheath block (RSB) analgesia: A randomised trial of patients with cancer and benign disease. Anticancer Res 39: 6751-6757, 2019. PMID: 31810940. DOI: 10.21873 /anticanres. 13890
18 Kärkkäinen J, Selander T, Purdy M, Juvonen P and Eskelinen M: Patients with increased levels of the oxidative stress biomarker SOD1 appear to have dimished postoperative pain after midline laparotomy: A randomized trial with special reference to postoperative pain score (NRS). Anticancer Res 38: 1003-1008, 2018. PMID: 2937473. DOI: 10.21873/anticanres. 12315

19 Lu MC, Yang MD, Li PC, Fang HY, Huang HY, Chan YC and Bau DT: effect of oligomeric proanthocyanidin on the antioxidant status and lung function of patients with chronic obstructive pulmonary disease. In Vivo 32: 753-758, 2018. PMID: 29936455. DOI: 10.21873/invivo.11304

20 Bakirezer SD, Yaltirik CK, Kaya AH, Yilmaz SG, Ozdogan S, Billur D and Isbir T: The evaluation of glutathione reductase and malondialdehyde levels in patients with lumbar disc degeneration disease. In Vivo 33: 811-814, 2019. PMID: 31028201. DOI: 10.21873 /invivo. 11543

21 Dogaru G, Bulboaca A, Boarescu PM, Ciumarnean L, Rus V, Sitar-Taut AV, Munteanu C, Bodisz G and Stanescu I: The effect of mofettes on oxidative stress/antioxidant balance in experimental myocardial ischemia. In Vivo 33: 1911-1920, 2019. PMID: 31662519. DOI: 10.21873/invivo.11685

22 Tsounapi P, Honda M, Hikita K, Sofikitis N and Takenaka A: Oxidative stress alterations in the bladder of a short-period type 2 diabetes rat model: Antioxidant treatment can be beneficial for the bladder. In Vivo 33: 1819-1826, 2019. PMID: 31662508. DOI: 10.21873 /invivo.11674

23 Uchiyama H, Uehara K, Nagashima T, Nakata A, Sato K, Mihara Y, Komatsu KI, Takanari J, Shimizu S and Wakame K: Global liver gene expression analysis on a murine metabolic syndrome model treated by low-molecular-weight lychee fruit polyphenol (Oligonol ${ }^{\circledR}$ ). Anticancer Res 36: 3705-3713, 2016. PMID: 27354644.
Received December 2, 2019

Revised December 10, 2019

Accepted December 12, 2019 